\title{
On the Trade-off between Throughput Maximization and Energy Consumption Minimization in IEEE 802.11 WLANs
}

\author{
Pablo Serrano, Matthias Hollick and Albert Banchs
}

\begin{abstract}
Understanding and optimizing the energy consumption of wireless devices is critical to maximize the network lifetime and to provide guidelines for the design of new protocols and interfaces. In this work we first provide an accurate analysis of the energy performance of an IEEE 802.11 WLAN, and then we derive the configuration to optimize it. We further analyze the impact of the energy configuration of the stations on the throughput performance, and we discuss under which circumstances throughput and energy efficiency can be both jointly maximized and where they constitute different challenges. Our findings are that, although an energyoptimized configuration typically yields gains in terms of throughput as compared against the default configuration, it comes with a reduction in performance as compared against the maximumbandwidth configuration, a reduction that depends on the energy parameters of the wireless interface.
\end{abstract}

Index Terms: Energy efficiency, energy optimization, IEEE 802.11, throughput optimization

\section{INTRODUCTION}

Information and communication technology (ICT) holds one of the keys to the reduction of greenhouse gases produced worldwide. However, increasing the energy efficiency of computing as well as networking can also significantly reduce the consumption of energy in the ICT sector itself. The importance of "greening the Internet" is thus recognized as a primary design goal of future global network infrastructures. It is estimated that, today, the Internet already accounts for about $2 \%$ of total world energy consumption, and with the current trend of shifting offline services online, this percentage is expected to grow significantly in the next years. The energy consumption is to be further fueled by the forthcoming Internet-based platforms that require always-on connectivity.

However, communication protocols, and in particular the technologies used in the access network, have been originally conceived to optimize metrics other than energy, such as throughput or delay. Greening these protocols thus represents a shift in the design paradigm, where energy instead of time is the most critical network resource. We no longer want to maximize the bits sent per time unit, but instead the bits the network can

Manuscript received XXX; approved for publication by XXX, XXX, XXX.

P. Serrano and A. Banchs are with the Departament of Ingeniería Telemática, Universidad Carlos III de Madrid, Avda. Universidad 30, 28911 Leganés, Spain, email: \{pablo,banchs\}@it.uc3m.es.

M. Hollick is with the Secure Mobile Networking Lab, Technische Universität Darmstadt, Mornewegstr. 32, 64293 Darmstadt, Germany, email: matthias.hollick@cased.de

A. Banchs is also with IMDEA Networks, Avda. Mar Mediterráneo 22, 28912 Leganés, Spain send per each joule consumed. Still, it is intuitively clear that this will not come for free, and there might be a price to pay in terms of throughput performance when developing sustainable and energy efficient architectures.

In this paper we assess to which extent the (old) throughput maximization and the (new) energy-efficiency maximization objectives diverge, for the case of IEEE 802.11 WLANs. Previous work has solved the configuration of WLANs for throughput maximization, starting from the statical approaches of e.g. [1,2] and including later adaptive approaches to maximize the bits per second sent e.g. $[3,4]$. However, from the point of view of energy consumption, most of the research so far has addressed the analytical or experimental characterization of the energy consumption of the WLAN [5-7], which is typically divided in three states: transmission, reception and idle-state (see Table 1 for the energy consumption of selected wireless network cards as well as two synthetic energy profiles to explore the parameter space for future wireless network cards). There has been also some proposals for energy efficiency optimization (e.g. [8-10]), typically based on heuristics and sometimes requiring changes to the MAC layer. However, these approaches did not discuss nor assess the extent to which the objective of energy optimization significantly differed from the classical throughput maximization objective.

To the best of our knowledge, only Bruno et al. [11] have considered the relation between throughput and energy and have discussed whether these could be both jointly maximized or not. However, their model consisted of a $p$-persistent CSMAbased WLAN, where interfaces only consumed energy in two states (transmission and reception), instead of the three states described above, i.e., they neglected the energy consumed during the idle state. In their model, consisting of a p-persistent CSMA-based WLAN where interfaces only consumed energy in two states (transmission and reception), it was shown that energy efficiency and throughput do not constitute different objectives and can jointly maximized. In this paper, where we improve the accuracy of the energy consumption model, we prove that this is not always the case.

The rest of the paper is organized as follows. In Section II we present and validate an analytical model of the energy consumption of a WLAN. We further introduce a new approximate model that trades off model accuracy for the sake of simplicity (nevertheless, as shown in the validation part, this reduction of accuracy is negligible, in particular in the region of optimal performance). Section III presents the two approaches for performance maximization: the throughput-based approach of Bianchi [1], and our energy-based approach that builds upon the approx- 
Table 1. Power consumption in Watts for different wireless interfaces (as reported in [8] and extended for synthetic power profiles)

\begin{tabular}{|l|l|r|r|r|r|}
\hline$\#$ & Card & $\rho_{t}$ & $\rho_{r}$ & $\rho_{i}$ & $\rho_{r} / \rho_{i}$ \\
\hline A & Lucent WaveLan & 1.650 & 1.400 & 1.150 & 1.22 \\
B & SoketCom CF & 0.924 & 0.594 & 0.066 & 9.00 \\
C & Intel PRO 2200 & 1.450 & 0.850 & 0.080 & 10.63 \\
D & Synthetic 1 & 1.450 & 0.850 & 0.170 & 5.00 \\
E & Synthetic 2 & 1.450 & 0.850 & 0.043 & 20.00 \\
\hline
\end{tabular}

imate analysis to derive a closed-form expression for the optimal transmission probability. In Section IV we compare the resulting configuration and performance from each approach, and discuss in which circumstances energy and throughput constitute different maximization objectives. Finally, Section V concludes the paper.

\section{ENERGY CONSUMPTION ANALYSIS}

Our analytical model for the energy consumption of a WLAN requires the following input parameters: $N$, the number of stations in the WLAN; $C W_{\min }$, defined as the minimum contention window stations use on their first attempt; and $\left\{\rho_{t}, \rho_{r}, \rho_{i}\right\}$, defined as the power consumed by the wireless interfaces when transmitting, receiving and idling, respectively. We assume all stations have always a packet of fixed length $L$ ready for transmission ${ }^{1}$, i.e., the network operates under saturation conditions, and that the sole reason for frame loss is a collision (where two or more stations transmit simultaneously). We further assume that each station randomly selects the destination for each frame out of the other $N-1$ stations.

\section{A. MODEL}

With the assumption that each transmission attempt collides with a constant and independent probability, we can model the behavior of a station with the same Markov chain used in [1]. Then, the probability that a station operating under saturation conditions transmits upon a backoff counter decrement $\tau$ can be computed by means of the following equation given by [1]

$$
\tau=\frac{2}{1+C W_{\min }+p C W_{\min } \sum_{i=0}^{m-1}(2 p)^{i}}
$$

where $p$ is the probability that a transmission attempt of a station collides. This probability can be computed as

$$
p=1-(1-\tau)^{N-1}
$$

The above constitutes a system of two non-linear equations that can be solved numerically, giving the value for $\tau$. With this, we next proceed to compute the energy per slot consumed by a station, which we denote by $e$.

We compute $e$ by applying the total probability theorem as follows:

$$
e=\sum_{j \in \Theta} E(j) p(j)
$$

\footnotetext{
${ }^{1}$ Note that, following our analysis of [12], the model could be extended to account for variable packet sizes.
}

where $\Theta$ is the set of events that can take place in a single times$\operatorname{lot}^{2}$, while $E(j)$ and $p(j)$ are the energy consumed in case of event $j$ and its probability, respectively. The set $\Theta$ of events, as well as their probabilities, is listed as follows:

- The slot is empty, $p_{e}$

- There is a success from the considered station, $p_{s, i}$

- There is a success from another station, $p_{s, \neg i}$

- There is a collision and the considered station is involved, $p_{c, i}$

- There is a collision but the considered station is not involved, $p_{c, \neg i}$

This way we can expand (1) as follows:

$$
\begin{aligned}
e & =p_{e} \rho_{i} T_{e}+ \\
& +p_{s, i}\left(\rho_{t} T_{s}+\rho_{r} T_{a c k}+\rho_{i}(\text { SIFS }+ \text { DIFS })\right)+ \\
& +p_{s, \neg i}\left[\rho_{r} T_{s}+\frac{1}{N-1}\left(\rho_{t} T_{a c k}\right)+\right. \\
& \left.+\frac{N-2}{N-1}\left(\rho_{r} T_{a c k}\right)+\rho_{i}(S I F S+D I F S)\right]+ \\
& +p_{c, i}\left(\rho_{t} T_{s}+\rho_{i} E I F S\right)+p_{c, \neg i}\left(\rho_{r} T_{s}+\rho_{i} E I F S\right)
\end{aligned}
$$

where $T_{e}, T_{s}$, and $T_{a c k}$ are the durations of an empty slot, a successful transmission and the transmission of an acknowledgment, while $S I F S, D I F S$, and EIFS are physical constants (for the computation of these values, see e.g. [1]).

The probability of each event can be easily computed based on the probability of a transmission $\tau$ as follows

$$
\begin{gathered}
p_{e}=(1-\tau)^{N} \\
p_{s}=N \tau(1-\tau)^{N-1} \\
p_{s, i}=\tau(1-\tau)^{N-1} \\
p_{s, \neg i}=p_{s}-p_{s, i} \\
p_{c}=1-p_{e}-p_{s} \\
p_{c, i}=\tau\left(1-(1-\tau)^{N-1}\right) \\
p_{c, \neg i}=p_{c}-p_{c, i}
\end{gathered}
$$

However, note that the full expression of (1) consists of a sum of several terms that non-linearly depends on $\tau$. In order to derive the value of $\tau$ that provides the best energy performance, we introduce the following simplified expression for $e$, which we denote by $\hat{e}$

$\hat{e}=(1-\tau)^{N} \rho_{i} T_{e}+\tau \rho_{t} T_{s}+(1-\tau)\left(1-(1-\tau)^{N-1}\right) \rho_{r} T_{s}$

This way, we have simplified the set $\Theta$ of events by considering only three cases:

1. nobody transmits,

2. the station transmits (without the distinction if there is a collision or a success), and

3. some other station transmits (again, no matter if there is a success of a collision).

The above can be expressed as:

$$
\hat{e}=R+\tau(T-R)-(1-\tau)^{N}(R-E)
$$

${ }^{2}$ A timeslot is defined as the amount of time between two backoff counter decrements of a station, see [1]. 


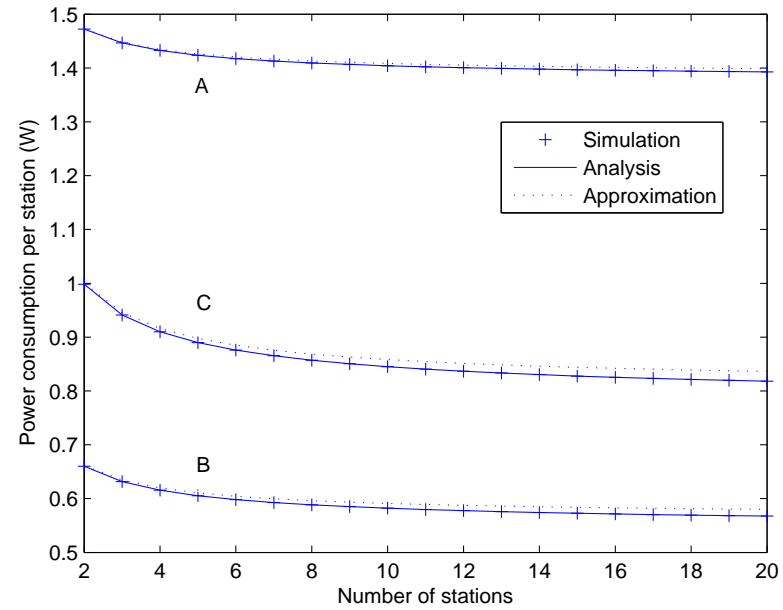

Fig. 1. Power consumption for the energy profiles $A, B$ and $C$ from Table 1

where $E=\rho_{i} T_{e}, T=\rho_{t} T_{s}$, and $R=\rho_{r} T_{s}$. We further write $T^{\prime}=T-R$ and $R^{\prime}=R-E$, therefore:

$$
\hat{e}=R+\tau T^{\prime}-(1-\tau)^{N} R^{\prime}
$$

With the above, the power consumption $\pi$ can be derived by dividing the average energy consumed per slot time over the average duration of a slot, i.e.,

$$
\pi=\frac{e}{T_{\text {slot }}}
$$

where $T_{\text {slot }}$ is given by

$$
T_{\text {slot }}=p_{e} T_{e}+\left(1-p_{s}-p_{c}\right) T_{s}
$$

Finally, we define the energy efficiency $\eta$ as the ratio between the bits transmitted and the energy consumed in a timeslot:

$$
\eta=\frac{p_{s, i} L}{e}
$$

\section{B. VALIDATION}

We first compare the accuracy of the exact and approximate models for $e$ and $\hat{e}$ against results obtained via simulation. Simulations are performed with an event-driven simulator developed by us, that closely follows the 802.11 DCF protocol details for each independently transmitting station, taking as input the number of stations $N$ and the set of $\{\rho\}$ parameters to use, and provides as output the total throughput and power consumed in the WLAN. We compare the energy consumed per second $\pi$ for the three selected power consumption sets A, B and C listed in Table 1 for different values of $N$ and the default DCF configuration (note that we do not show the results from synthetic interfaces in Figs.1-3 and Table 2 for ease of interpretation of the plots, these will be considered in the energy optimization below). We model the physical layer with the parameters of the IEEE 802.11b standard. Results are shown in Fig. 1.

From the results, it is clear that the detailed analytical model $e$ provides values that almost coincide with those derived from

\begin{tabular}{|c|c|c|c|c|c|}
\hline Card & $C W_{\min }$ & $N$ & $\operatorname{Sim}(\mathrm{W})$ & Model (W) & Error $(\%)$ \\
\hline \multirow[t]{3}{*}{$\mathrm{A}$} & \multirow[t]{3}{*}{8} & 5 & 1.4237 & 1.4263 & 0.1843 \\
\hline & & 10 & 1.4042 & 1.4086 & 0.3082 \\
\hline & & 20 & 1.3927 & 1.3987 & 0.4311 \\
\hline & \multirow[t]{3}{*}{32} & 5 & 1.4237 & 1.4263 & 0.1843 \\
\hline & & 10 & 1.4042 & 1.4086 & 0.3082 \\
\hline & & 20 & 1.3927 & 1.3987 & 0.4311 \\
\hline & \multirow[t]{3}{*}{128} & 5 & 1.4237 & 1.4263 & 0.1843 \\
\hline & & 10 & 1.4042 & 1.4086 & 0.3082 \\
\hline & & 20 & 1.3927 & 1.3987 & 0.4311 \\
\hline \multirow[t]{9}{*}{$\mathrm{B}$} & \multirow[t]{3}{*}{8} & 5 & 0.6054 & 0.6107 & 0.8713 \\
\hline & & 10 & 0.5821 & 0.5910 & 1.5224 \\
\hline & & 20 & 0.5675 & 0.5800 & 2.1971 \\
\hline & \multirow[t]{3}{*}{32} & 5 & 0.6054 & 0.6107 & 0.8713 \\
\hline & & 10 & 0.5821 & 0.5910 & 1.5224 \\
\hline & & 20 & 0.5675 & 0.5800 & 2.1971 \\
\hline & \multirow[t]{3}{*}{128} & 5 & 0.6054 & 0.6107 & 0.8713 \\
\hline & & 10 & 0.5821 & 0.5910 & 1.5224 \\
\hline & & 20 & 0.5675 & 0.5800 & 2.1971 \\
\hline \multirow[t]{9}{*}{$\mathrm{C}$} & \multirow[t]{3}{*}{8} & 5 & 0.8898 & 0.8977 & 0.8826 \\
\hline & & 10 & 0.8452 & 0.8583 & 1.5488 \\
\hline & & 20 & 0.8181 & 0.8364 & 2.2384 \\
\hline & \multirow[t]{3}{*}{32} & 5 & 0.8898 & 0.8977 & 0.8826 \\
\hline & & 10 & 0.8452 & 0.8583 & 1.5488 \\
\hline & & 20 & 0.8181 & 0.8364 & 2.2384 \\
\hline & \multirow[t]{3}{*}{128} & 5 & 0.8898 & 0.8977 & 0.8826 \\
\hline & & 10 & 0.8452 & 0.8583 & 1.5488 \\
\hline & & 20 & 0.8181 & 0.8364 & 2.2384 \\
\hline
\end{tabular}
simulations, while the approximate model $\hat{e}$ follows very closely
Table 2. Power consumption per interface for different wireless interfaces

the behavior of the WLAN but slightly overestimating the energy consumed for large values of $N$. This overestimation is caused because, in the simplified model, transmission attempts are assumed successful in all cases, which requires more energy (because an acknowledgment has to be sent and received) than collisions.

We further validate the accuracy of the approximate model for a wider range of $C W_{\min }$ and $N$ values, for the interfaces A, B, and C of Table 1. Results are shown in Table 2. We note that the relative error is well below $5 \%$ in all cases, and only approximates $2.5 \%$ when the number of stations is relatively large $(N=20)$, due to the overestimation of the energy wasted during collisions.

We take advantage of the accurate analytical model to further explore the energy consumption of the WLAN, identifying where the energy consumption is rooted. To this aim, we obtain the relative amount of energy wasted during empty slots or collisions for a varying number of stations, with the results of Fig. 2 for interfaces $\mathrm{A}$ and $\mathrm{C}$ of Table 1.

It can be seen from the figure that, as $N$ increases, so do collisions and more and more energy is wasted in unsuccessful transmissions. On the other hand, for the case of interface A, when $N$ is relatively small $(N \leq 4)$ more than $5 \%$ of the energy is spend in backoff counter decrements during empty timeslots, a result caused by the overly large values of $C W_{\min }$ for these number of stations. However, note that while this energy wasted in idling is 

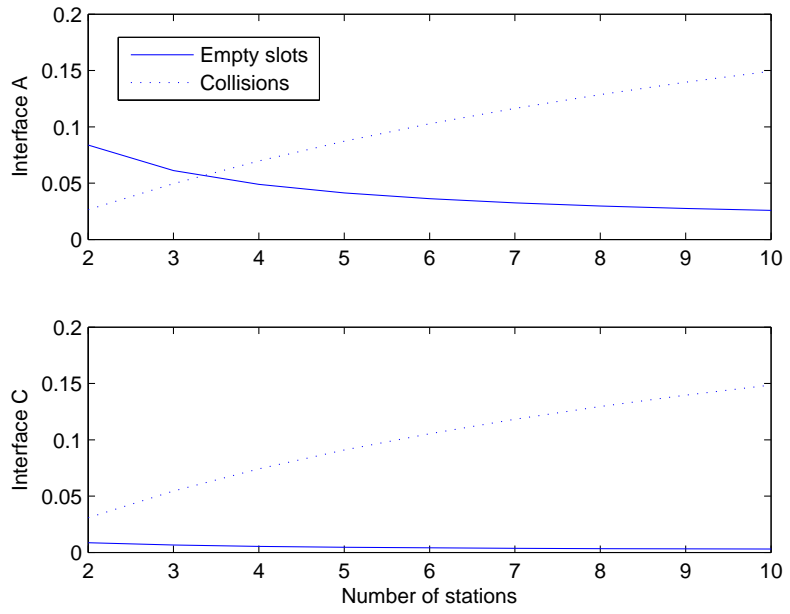

Fig. 2. Relative energy devoted to collisions and idling for the selected energy profiles $\mathrm{A}$ and $\mathrm{C}$ from Table 1

non-negligible for the case of interface A (in fact, is larger than the energy wasted in collisions), for the case of $\mathrm{C}$ this is never the case: the relative energy wasted in idling never reaches $1 \%$.

This behavior can be intuitively explained if we consider that the cost of a timeslot that contains a collision or is empty, in fact, "multiplied" by the power consumed when transmitting/receiving or idling, respectively. Therefore, for the case of interface $\mathrm{C}$, the relative small duration of an empty timeslot is further multiplied by $\rho_{i}$, which is an order of magnitude below $\rho_{r}$ and $\rho_{t}$ and results in a negligible contribution to the total energy consumption. For the case of interface $\mathrm{A}$, as the ratio $\rho_{r} / \rho_{i}$ is not that large, the energy wasted in idling can be compared to the energy wasted during collisions for small values of $N$.

Next we compare the efficiency $\eta$ for three different WLAN scenarios (one for each of the first three interfaces of Table 1) and the standard recommended configuration of $C W_{\min }$. To this aim, we plot in Fig. 3 the value of $\eta$ given by simulations against the ones provided by the accurate analytical model of (3), and the simplified model, i.e., using (3) but substituting $e$ with $\hat{e}$.

From the figure, is evident that the default recommendation shows an efficiency $\eta$ that rapidly decreases with the number of stations $N$, a result expected because of the increasing number of collisions. To analyze the impact of the $C W_{\min }$ value used on $\eta$, in Fig. 4 we plot the energy efficiency of the WLAN for different values of the initial contention window, using the five energy profiles of Table $1^{3}$. We show results from the simulations and using our approximated model $\hat{e}$, and we also mark with a triangle the maximum value of $\eta$ obtained.

From the figure, we can see two main results: first, the model is very accurate, in particular in the region where the maximum value of $\eta$ is reached ${ }^{4}$; second, this maximum value of $\eta$ is ob-

\footnotetext{
${ }^{3}$ Note that profiles A-C are taken from [8], while the two additional profiles D and $\mathrm{E}$ are used to represent interfaces with the same transmission and reception power as of $\mathrm{C}$, but different power consumption when idling-we argue that this is the parameter most likely to change in modern interfaces.

${ }^{4}$ Note that, for the considered $C W_{\min }$ values and $N=10$ stations, the efficiency of all interfaces but A shows a relatively flat figure. However, for larger $C W_{\min }$ values the efficiency would still drop to 0 , as stations would
}

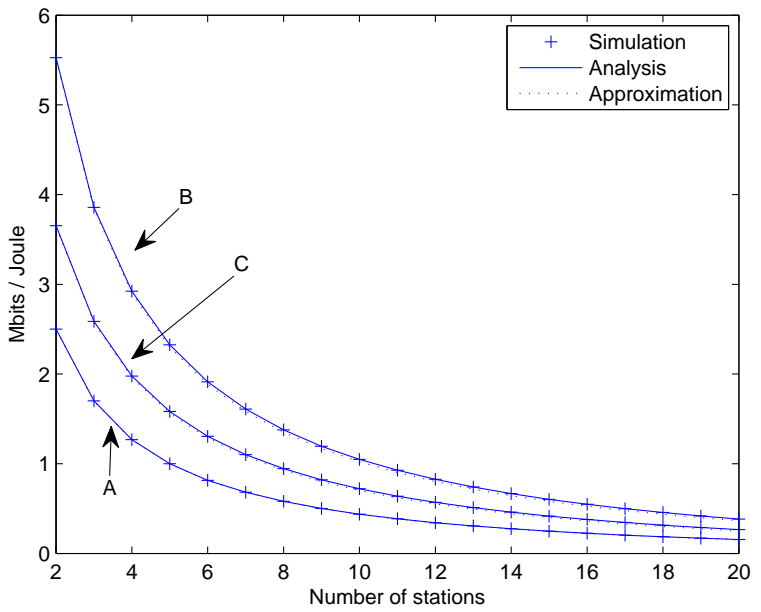

Fig. 3. Impact of the $C W_{\min }$ used on the energy efficiency for the selected energy profiles (A), (B), and (C) from Table 1

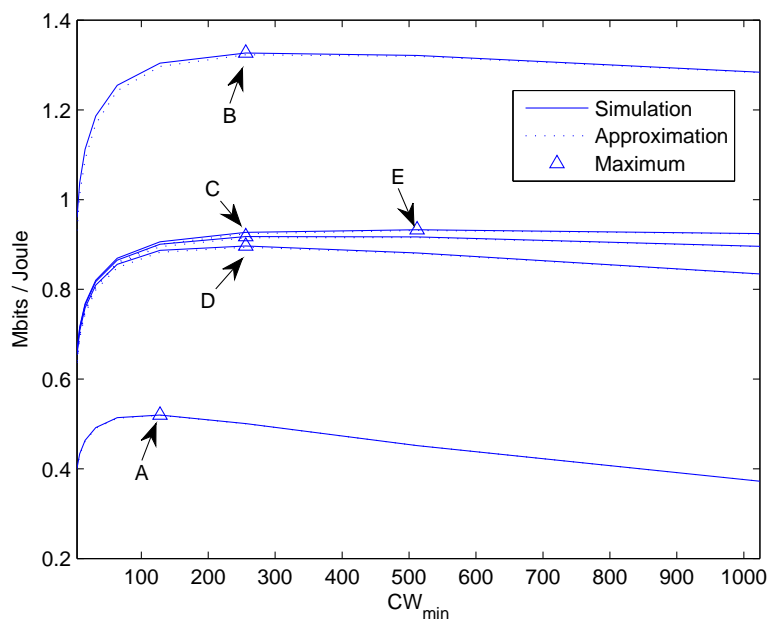

Fig. 4. Energy efficiency of the WLAN for $N=10$ stations and the interfaces of Table 1

tained for different $C W_{\min }$ values, depending on the WLAN interface considered. Note that this observation is aligned with the results obtained in Fig. 2, where the relative costs of idling and colliding are multiplied by different values depending on the interface. This way, the $C W_{\min }$ value that achieves best performance is larger when the $\rho_{i}$ value is relatively smaller, as colliding is riskier than using larger values of the contention window. This relation between the power consumption parameters and the optimal value of $C W_{\min }$ is analyzed in the next section.

\section{CONFIGURATION OF 802.11: THROUGHPUT-BASED VS. ENERGY-BASED}

We provide in this section closed-form expressions for the optimal transmission probability $\tau$, depending on the optimization objective: throughput maximization in Section III-A, and energy optimization in Section III-B. Note that, to derive a con-

spend most of the time in backoff counter decrements. 
figuration rule, if we set $C W_{\min }=C W_{\max }$, the transmission probability $\tau$ is related to the size of the contention window $C W$ to be used as follows

$$
C W=\frac{2}{\tau}-1
$$

and therefore it would be immediate, for an Access Point implementing the EDCA mechanism of the IEEE 802.11e standard, to broadcast the $C W$ value to use once computed the optimal $\tau$ value as given in the next sections.

\section{A. THROUGHPUT MAXIMIZATION}

When optimizing throughput, it is well known that CSMA/CA algorithms have an optimal transmission probability that depends on the network load, in terms of traffic generated and number of contending stations. For the case of saturated IEEE 802.11 WLANs, Bianchi [1] analytical derived the optimal transmission probability $\tau$ that maximizes throughput, where throughput is computed as the average payload transmitted in a slot time over the average slot duration, i.e.,

$$
R=\frac{p_{s} L}{T_{\text {slot }}}
$$

where $L$ is the frame size, $p_{s}$ is the probability that a slot contains a successful transmission and $T_{\text {slot }}$ is the average slot duration (note that these two probabilities were already computed in the previous section).

This optimization is done by deriving the above with respect to $\tau$, and solving a second-grade equation resulting from the approximation $\tau \ll 1$. This results in the following approximate value for the optimal transmission probability that maximizes throughput, which we denote by $\tau_{t}$

$$
\tau_{t} \approx \frac{1}{N} \sqrt{\frac{2 T_{e}}{T_{s}}}
$$

Note that this optimal value of $\tau$ depends on the number of stations $N$, but also on the relative size of an empty timeslot $T_{e}$ as compared to a timeslot that contains a transmission $T_{s}$. This way, apart from the number of stations, the ratio between the timeslot lengths sets the optimal tradeoff between the cost of a collision and the cost of idling. Indeed, this is the motivation behind some adaptive algorithms (e.g. Idle Sense [3]) that equalize the amount of time wasted in collisions with the amount of time waiting in backoff decrements.

However, because $\tau_{t}$ does not take into account energy consumption, for similar scenarios with different WLAN interfaces it will provide the same configuration for $C W$, while we have seen in Fig. 3 that the optimal CW value indeed depends on the energy consumption of the WLAN interfaces. This relationship is what we analyze next.

\section{B. ENERGY OPTIMIZATION}

To compute the transmission probability that optimizes the consumption of energy $\tau_{e}$ we start from the expression of $\eta$ with the approximation for $\hat{e}$

$$
\eta=\frac{\tau(1-\tau)^{N-1} L}{R+\tau T^{\prime}-(1-\tau)^{N} R^{\prime}}
$$

And then compute the $\tau$ value that maximizes the above by

$$
\frac{d \eta}{d \tau}=0
$$

This leads to

$$
(N-1) \tau^{2} T^{\prime}+(1-\tau)^{N} R^{\prime}+N \tau R-R=0
$$

By the following Taylor expansion of $(1-\tau)^{n}$

$$
(1-\tau)^{N} \approx 1-n \tau+\frac{1}{2} N(N-1) \tau^{2}
$$

We have the following equation

$$
a \tau^{2}+b \tau+c=0
$$

where

$$
\begin{gathered}
a=(N-1) T^{\prime}+\frac{1}{2} N(N-1) R^{\prime} \\
b=N E \\
c=-R
\end{gathered}
$$

If we now define $\alpha$ and $\beta$ as follows

$$
\alpha=\frac{T^{\prime}}{E}, \beta=\frac{R^{\prime}}{E}
$$

Then we have the following for the computation of $\tau_{e}$ :

$$
\tau_{e}=\frac{-N+\sqrt{N^{2}+4(N-1) \alpha+2 N(N-1) \beta}}{2(N-1) \alpha+N(N-1) \beta}
$$

Which can be approximated as follows

$$
\tau_{e} \approx \frac{1}{N} \sqrt{\frac{2}{\beta}} \approx \frac{1}{N} \sqrt{\frac{2 \rho_{i} T_{e}}{\rho_{r} T_{s}}}
$$

We validate this expression for the interfaces of Table 1 and different values of $N$ in Table 3. To this aim, we compute the $C W$ value that provides the best performance $C W_{e x}$, the $C W$ value derived from the use of (5), $C W_{\text {conf }}$, and the energy efficiency resulting from each case, $\eta_{e x}$ and $\eta_{\text {conf }}$, respectively.

For all scenarios considered, the resulting $C W$ values are relatively close, although for large $N$ values there is a larger difference because of the larger $C W$ values needed. Still, the resulting values of $\eta$ are almost identical in all cases, a result caused by the "flatness" of $\eta$ in the region close to the maximum value. From the results of the table, we conclude that indeed (5) provides the most energy-efficient configuration to use in a WLAN.

To sum up, the optimal value of $\tau$ for this case depends not only on the relative size of the timeslots, like in the case of throughput maximization, but also on the relative power consumed when receiving or idling. This way, for old interfaces where idling and receiving consumes approximately the same value (e.g., interface A of Table 1) the formula of Bianchi still holds, while for new interfaces where the cost of idling is smaller (e.g., interface $\mathrm{C}$ ), the $C W_{\min }$ value to use is larger than the one obtained with (4). Actually, if we divide (4) by (5), the relation between $\tau_{t}$ and $\tau_{e}$ is given by the ratio of the power consumption of the interface when receiving a frame over the power consumption when idling, i.e.,

$$
\frac{\tau_{t}}{\tau_{e}}=\sqrt{\rho_{r} / \rho_{i}}
$$

a relation that we will further analyze and discuss in the next Section. 
Table 3. Comparison between the exhaustive search and the configuration algorithm in kbits/J

\begin{tabular}{|r|r|r|r|r|r|}
\hline Card & $N$ & $C W_{e x}$ & $C W_{\text {conf }}$ & $\eta_{e x}$ & $\eta_{\text {conf }}$ \\
\hline A & 5 & 64 & 65 & 1.0317 & 1.0316 \\
& 10 & 132 & 131 & 0.5204 & 0.5204 \\
& 20 & 285 & 262 & 0.2613 & 0.2614 \\
\hline B & 5 & 166 & 178 & 2.5325 & 2.5323 \\
& 10 & 332 & 357 & 1.3285 & 1.3284 \\
& 20 & 666 & 715 & 0.6811 & 0.6811 \\
\hline C & 5 & 181 & 193 & 1.7311 & 1.7310 \\
& 10 & 399 & 388 & 0.9196 & 0.9197 \\
& 20 & 665 & 777 & 0.4747 & 0.4748 \\
\hline D & 5 & 132 & 132 & 1.6906 & 1.6906 \\
& 10 & 249 & 266 & 0.8967 & 0.8967 \\
& 20 & 499 & 533 & 0.4626 & 0.4626 \\
\hline E & 5 & 249 & 266 & 1.7544 & 1.7543 \\
& 10 & 499 & 533 & 0.9329 & 0.9329 \\
& 20 & 999 & 1066 & 0.4819 & 0.4818 \\
\hline
\end{tabular}
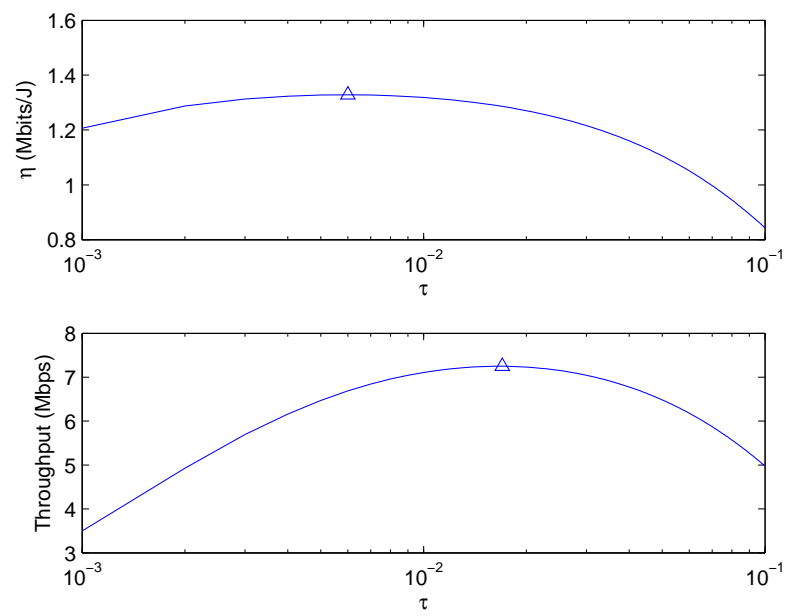

Fig. 5. Energy efficiency and Throughput vs $\tau$

\section{ENERGY-CONSUMPTION MINIMIZATION VS. THROUGHPUT MAXIMIZATION}

Throughput maximization and energy efficiency optimization aim at different objectives. To illustrate this, we plot in Fig. 5 the throughput and energy efficiency achieved for the case of $N=$ 10 , with the interface $\mathrm{C}$ of Table 1 , and using different values of the transmission probability $\tau$. Note that the value of $\tau$ that achieves optimal throughput is approximately 0.006 , while the value that maximizes energy efficiency is around 0.020 . If we divide these, the ratio is approximately the one obtained with the square root of the relative power consumption given in Table 1 , i.e., $\sqrt{\rho_{r} / \rho_{i}}=\sqrt{10} \approx 3.16$.

We have therefore proved that there is a different configuration for $C W_{\min }$ depending on the variable (i.e. throughput or energy) to optimize. We next compare the resulting configuration obtained when maximizing throughput and when maximizing energy efficiency. To this end, in Fig. 6 we show the resulting configuration of $C W$ for each maximization variable, for the interfaces of Table 1 and an increasing number of stations

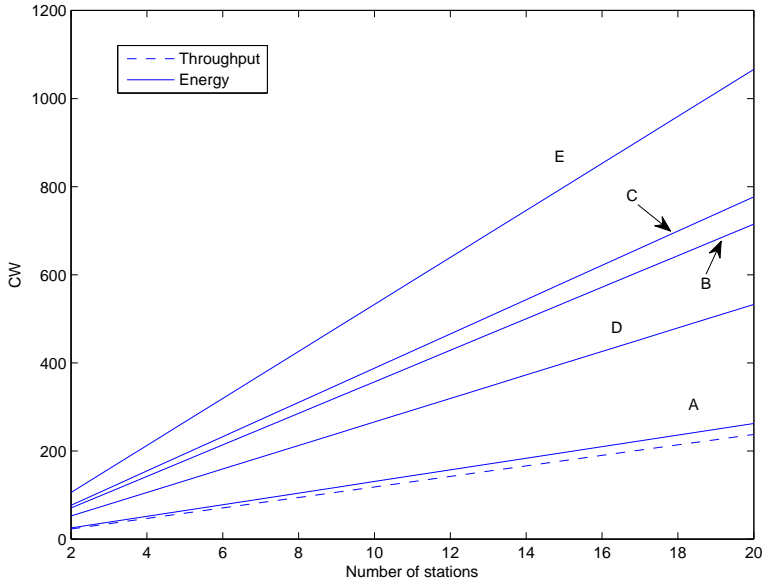

Fig. 6. Resulting $C W$ configuration from each approach

$N$. From the figure is obvious to see that, while the throughput maximization provides the same $C W$ for a given number of stations, the optimal $C W$ for energy efficiency depends noticeably on the power characteristics of the WLAN interface. It can be seen that, the larger the $\rho_{r} / \rho_{i}$ ratio, the larger the $C W$, as collisions have a larger cost and therefore it is more efficient to spend more time on the backoff, instead of taking the risk of transmitting and suffering from an energy-consuming collision that is unproductive in terms of throughput maximization.

We next compare against the performance of the throughput optimization and the energy optimization approaches. To this aim, we first compare them in terms of energy efficiency in Fig. 7, with a zoomed version provided in Fig. 8 (for a more complete comparison, we also include the performance of the default DCF configuration).

From the figures, is clear that both throughput and energy optimizing approaches substantially outperform the DCF default configuration in terms of energy efficiency, the later providing the best results: while for the case of interface A of Table 1 the differences are negligible, for the rest of the cases there is indeed an improvement when using $\tau_{e}$ instead of $\tau_{t}$. Apart from this improvement, that seems to depend on the absolute $\rho_{r}$ value, there is another result worth highlighting: despite the resulting $C W_{\text {min }}$ configuration for interfaces $\mathrm{C}, \mathrm{D}$ and $\mathrm{E}$ is different, as shown in Figure 6, the obtained $\eta$ values are very similar as seen in Fig. 8. This is because, even for an ideal case with no collisions or idling, the $\rho_{t}$ and $\rho_{r}$ values are the same for the three interfaces.

We next analyze the throughput performance provided by each configuration. To this aim, we plot in Fig. 9 the total throughput in the WLAN for different values of $N$. It is clear that the use of $\tau_{t}$ provides the largest values of throughput, as expected, these being very close to the ones provided by the maximum energy efficiency configuration when using interface A of Table 1 (because of the relative values of the $\rho$ parameters). However, for the rest of the interfaces, indeed there is a price to pay in terms of throughput when optimizing energy consumption, this price being larger the larger the ratio $\sqrt{\rho_{r} / \rho_{i}}$ is. Indeed, with an increasing value of this ratio the $C W_{\min }$ 


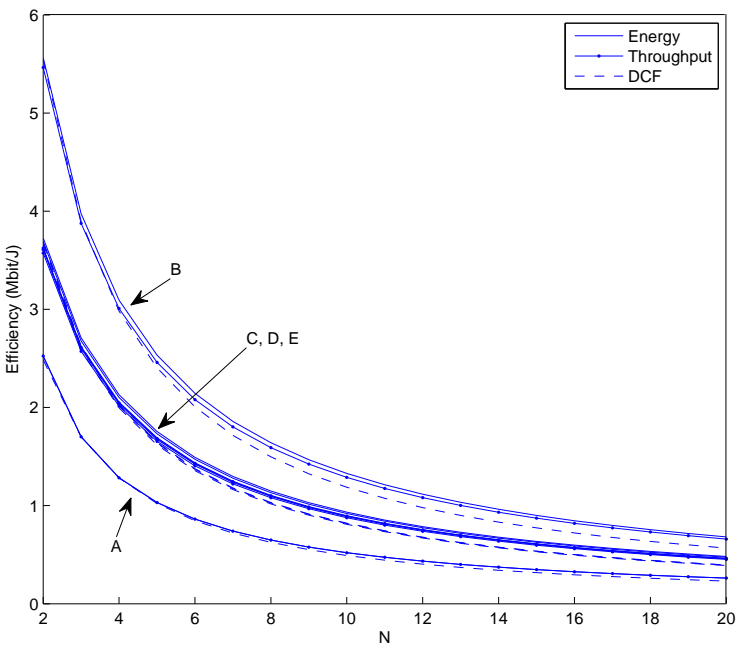

Fig. 7. Energy efficiency of each approach

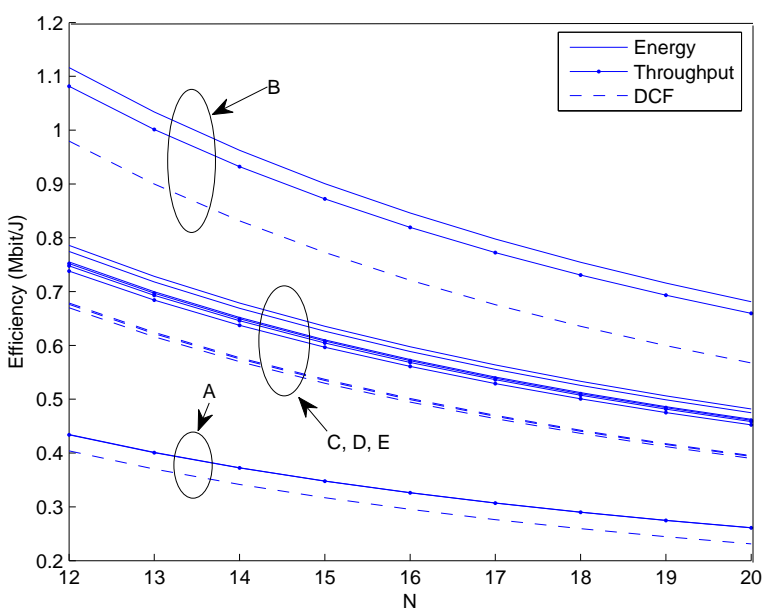

Fig. 8. Zoomed version of Fig. 7, for $N \in\{12,20\}$

of each approach diverge, as explained in the previous section, and therefore throughput and energy will not constitute the same maximization objective. Actually, the throughput obtained with the $\mathrm{B}, \mathrm{C}, \mathrm{D}$ and $\mathrm{E}$ interfaces is smaller than the one provided by DCF for the smaller values of $N$. From a certain number of stations on, however, the energy-optimized approach also results in better throughput compared to the DCF configuration (here: $N \geq 10$ for interface $\mathrm{D}, N \geq 16$ for interface $\mathrm{B}$, and $N \geq 18$ for interface $C$ ). Please note, though, that for the cases where the DCF configuration outperforms the energy-optimized configuration in terms of throughput, this DCF configuration causes significantly higher energy costs due to the different values of energy spent in collisions and backoff counter decrements.

Therefore, these results confirm that there is a tradeoff between energy and throughput maximization, which depends on the characteristics of the WLAN interface. Indeed, for some ratios of power consumption we have the same result of [11], that both throughput and energy efficiency can be simultaneously

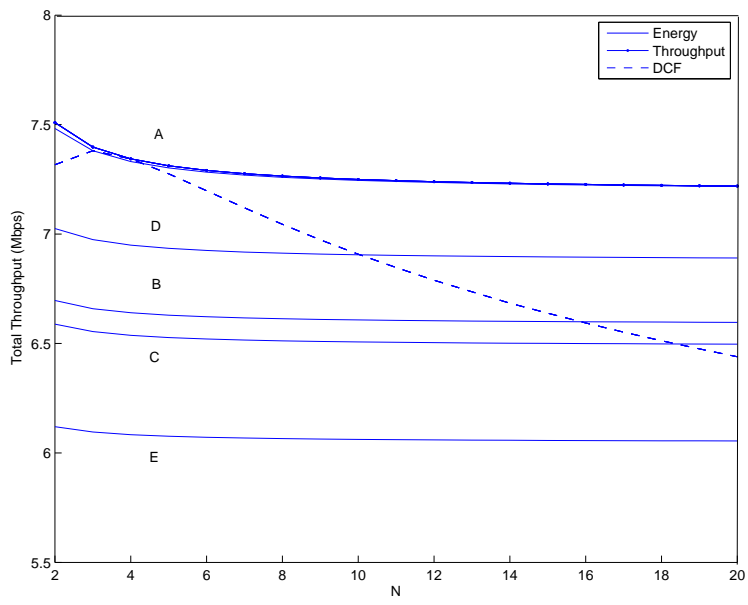

Fig. 9. Throughput performance of each approach. We show all energy profiles from Table 1

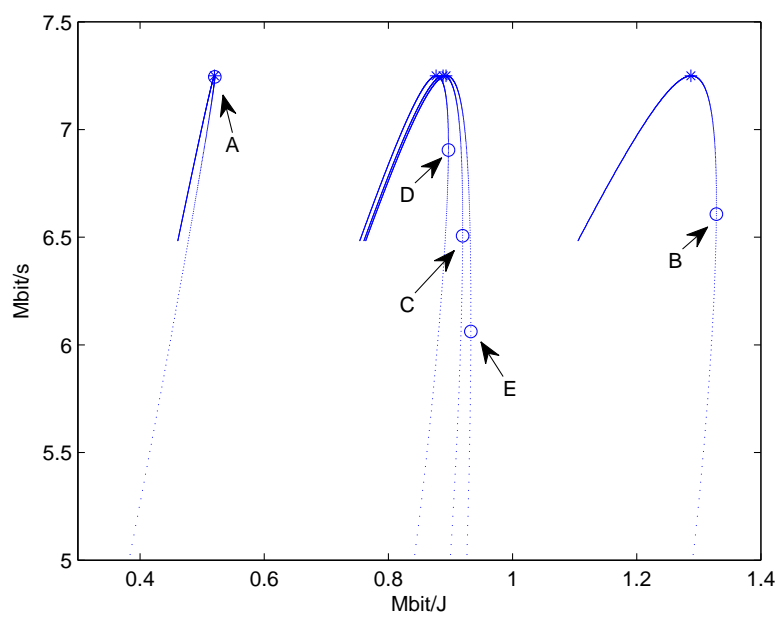

Fig. 10. Trade-off imposed by the energy features of the WLAN interfaces

maximized. However, our results show also that, for existing WLAN interfaces, this is not always the case.

This finding is summarized in Fig. 10. In this figure, we plot for the case of $N=10$ the resulting values of the energy efficiency (in the x-axis) and throughput (in the y-axis) for all possible configurations of $C W_{\min }$, and all the interfaces of Table 1. In the figure, we mark with a star the point of maximum throughput performance, and with a circle the point of maximum energy efficient. This figure provides valuable insights on the observed behavior:

- For the case of interface A, given its relatively similar values of power consumption $\rho_{t}, \rho_{r}$ and $\rho_{i}$, both energy and throughput can be jointly maximized, given the "linear" shape of the resulting curve.

- For the cases of interfaces B, C and D, the larger the $\rho_{r} / \rho_{i}$ ratio, the more separate the optimum values are and therefore the higher the price to pay in throughput when optimizing energy (and vice-versa). 
- Lastly, all curves are vertically aligned, because of the use of the physical layer parameters of $802.11 b^{5}$. However, their position in the X-axis is not tied to the $\rho_{i}$ parameter or the $\rho_{r} / \rho_{i}$ ratio, but instead is given by the other values of $\rho$.

This finding backs our above analysis and demonstrates that, depending on the power characteristics of the WLAN interfaces, gains w.r.t. energy are achievable if a energy-optimized configuration is chosen over a throughput-optimized one.

\section{CONCLUSIONS}

While the energy consumption was only considered a key performance figure in very specific environments (e.g., sensor networks), greening the communication protocols is nowadays recognized as a primary design goal of future global network infrastructures. This design goal requires switching from "information per unit of time" measurements to "information per unit of energy". However, for the case of 802.11 WLANs, energy optimization has been typically addressed through the use of ad-hoc sleeping heuristics, and not from the behavior of the CSMA/CA access mechanism.

The contribution of this paper is three-fold. First, we have revisited previous 802.11 performance analyses to derive an approximate model, analytically tractable, of the energy consumption of a 802.11 WLAN. Second, based on this approximate model, we have derived the optimal configuration to use to optimize energy performance. Third, based on this configuration we have discussed under which circumstances energy efficiency and throughput can be jointly maximized, and when they constitute different objectives.

\section{ACKNOWLEDGMENTS}

The research leading to these results has received funding from the European Community's Seventh Framework Programme (FP7/2007-2013) under grant agreement $n^{o} 214994$. The views and conclusions contained here are those of the authors and should not be interpreted as necessarily representing the official policies or endorsements, either express of the CARMEN project or the European Commission. It was also partly funded by the Ministry of Science and Innovation of Spain, under the QUARTET project (TIN2009-13992-C02-01).

\section{REFERENCES}

[1] G. Bianchi, Performance Analysis of the IEEE 802.11 Distributed Coordination Function, Selected Areas in Communications, IEEE Journal on, vol. 18, no. 3, pp. 535-547, Mar 2000.

[2] H. Wu, Y. Peng, K. Long, S. Cheng, and J. Ma, Performance of Reliable Transport Protocol over IEEE 802.11 Wireles LAN: Analysis and Enhancement, in Proceedings of IEEE INFOCOM '02, June 2002.

[3] M. Heusse, F. Rousseau, R. Guillier, and A. Duda, Idle Sense: An Optimal Access Method for High Throughput and Fairness in Rate Diverse Wireless LANs, SIGCOMM Comput. Commun. Rev., vol. 35, no. 4, pp. 121-132, 2005

[4] F. Cali, M. Conti, and E. Gregori, Dynamic Tuning of the IEEE 802.11 Protocol to Achieve a Theoretical Throughput Limit, IEEE/ACM Trans. Netw., vol. 8, no. 6, pp. 785-799, 2000.

[5] X. Wang, J. Yin, and D. P. Agrawal, Analysis and Optimization of the Energy Efficiency in the 802.11 DCF, Mob. Netw. Appl., vol. 11, no. 2, pp. 279-286, 2006.

\footnotetext{
${ }^{5}$ Therefore, the use of e.g. $802.11 \mathrm{~g}$ parameters would have shifted the curves to the 54 Mbps range.
}

[6] M. Ergen and P. Varaiya, Decomposition of Energy Consumption in IEEE 802.11, in Communications, 2007. ICC '07. IEEE International Conference on, June 2007, pp. 403-408.

[7] L. Feeney and M. Nilsson, Investigating the Energy Consumption of a Wireless Network Interface in an Ad Hoc Networking Environment, in Proceedings of IEEE INFOCOM '01, May 2001.

[8] V. Baiamonte and C.-F. Chiasserini, Saving Energy During Channel Contention in 802.11 WLANs, Mob. Netw. Appl., vol. 11, no. 2, pp. 287-296, 2006.

[9] J.-C. Chen and K.-W. Cheng, EDCA/CA: Enhancement of IEEE 802.11e EDCA by Contention Adaption for Energy Efficiency, Wireless Communications, IEEE Transactions on, vol. 7, no. 8, pp. 2866-2870, August 2008.

[10] C. Wang, B. Li, and L. Li, A New Collision Resolution Mechanism to Enhance the Performance of IEEE 802.11 DCF, Vehicular Technology, IEEE Transactions on, vol. 53, no. 4, pp. 1235-1246, July 2004.

[11] R. Bruno, M. Conti, and E. Gregori, Optimization of Efficiency and Energy Consumption in p-Persistent CSMA-based Wireless LANs, Mobile Computing, IEEE Transactions on, vol. 1, no. 1, pp. 10-31, Jan-Mar 2002.

[12] P. Serrano, A. Banchs, and A. Azcorra, A Throughput and Delay Model for IEEE 802.11e EDCA Under Non Saturation, Wirel. Pers. Commun., vol. 43, no. 2, pp. 467-479, 2007.

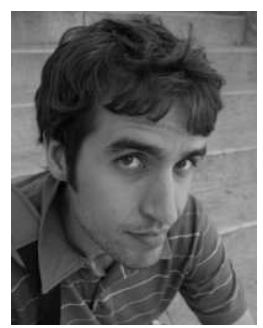

Dr. Pablo Serrano got his Telecommunication Engineering degree and his $\mathrm{PhD}$ from the University Carlos III of Madrid (UC3M) in 2002 and 2006, respectively. He has been with the Telematics Department of UC3M since 2002, where he currently holds the position of Assistant Professor. In 2007 he was a Visiting Researcher at the Computer Network Research Group at Univ. of Massachusetts Amherst partially supported by the Spanish Ministry of Education under a José Castillejo grant. He has over 30 scientific papers in peer-reviewed international journal and conferences. He also serves as TPC member of several international conferences, including IEEE Globecom and IEEE INFOCOM.

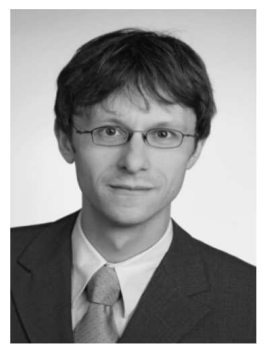

Dr. Matthias Hollick is heading the Secure Mobile Networking Lab (SEEMOO) at the Computer Science Department of Technische Universität Darmstadt, Germany. He received his doctoral degree in 2004 from the TU Darmstadt. His research focus is on secure and quality-of-service-aware communication for mobile and wireless ad hoc, mesh, and sensor networks. Dr. Hollick has been researching, working, and teaching at the TU Darmstadt, the Universidad Carlos III de Madrid (UC3M), and the University of Illinois at Urbana-Champaign (UILLINOIS). In 2005, for his research, Dr. Hollick has received the Adolf-Messer Foundation award.

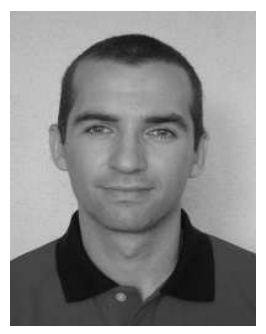

Dr. Albert Banchs received his Telecommunications Engineering degree from the Polytechnical University of Catalonia in 1997, and the $\mathrm{PhD}$ degree from the same university in 2002. He was a visitor researcher at ICSI, Berkeley, in 1997, worked for Telefónica I+D, in 1998, and for NEC Europe Ltd., Germany, from 1998 to 2003. Since 2003, he is with the University Carlos III of Madrid and since 2009 he is also Deputy Director of IMDEA Networks. A. Banchs authors over 50 publications in peer-reviewed journals and conferences and four patents (two of them granted). He is associated editor for IEEE Communications Letters and has been guest editor for IEEE Wireless Communications and Computer Networks. 